\title{
Gut Microbiota in NAFLD Pathogenesis and Possible Dietary-Based Strategies
}

\author{
Created by: Marica Meroni, MMiriam Longo , CPaola Dongiovanni \\ Version received: 24 April 2020
}

check for

updates

\begin{abstract}
Nonalcoholic fatty liver disease (NAFLD) is considered as a global healthcare burden since it is epidemiologically related to obesity, type 2 diabetes (T2D) and Metabolic Syndrome (MetS). It entails a broad spectrum of hepatic injuries, which includes simple and uncomplicated steatosis, nonalcoholic steatohepatitis (NASH), fibrosis, cirrhosis and hepatocellular carcinoma (HCC). The susceptibility to develop NAFLD is highly variable and it is influenced by several cues including environmental (i.e., dietary habits and physical activity) and inherited (i.e., genetic/epigenetic) risk factors. Emerging evidence has outlined the implication of intestinal microbiota and its harmful by-products as actively contributors to NAFLD pathophysiology, probably due to the tight anatomo-functional crosstalk between the gut and the liver. Unhealthy dietary habits may trigger qualitative and quantitative modifications in intestinal flora taxonomic composition, mucosal inflammation, and intestinal barrier derangement. An enhanced intestinal permeability, also referred to 'leaky gut', results in the translocation of viable pathogenic bacteria, Gram-negative products, and pro-inflammatory luminal metabolites into the bloodstream, further corroborating the liver damage. Furthermore, long-term dysbiosis may exert detrimental effect on hepatic epigenome. Indeed, epigenetic modifiers (DNA methylation, histone modifications and miRNAs regulation) are highly sensitive to environmental cues. Nowadays, no therapeutic interventions are available for NAFLD prevention and management, thus modifications in lifestyle, diet and physical exercise remain the cornerstone in therapeutic approaches to NAFLD, even in the light of their impact on intestinal microflora.
\end{abstract}

The human gastrointestinal lumen is the largest reservoir of microorganisms in the body, representing the physiological habitat for more than 100 trillion microorganisms (bacteria, archaea, fungi, yeast and viruses) [1]. Among them, $85 \%$ of total bacteria are commensal microbes that live in synergy with the host, providing biological and metabolic functions. The majority of bacteria belongs to the phyla Firmicutes (Gram positive) and to Bacterioidetes (Gram negative), mainly involved in the short-chain fatty acids (SCFAs), i.e., acetate, butyrate and propionate and hydrogen production, respectively[2][3][4]. Although the crucial role of intestinal flora remains under definition, growing evidence demonstrates that microbial species are directly implicated in the processing and digestion of complex and indigestible polysaccharides to SCFAs, guaranteeing the energy supply to the host and the intestinal barrier preservation. Indeed, in physiological conditions, gut microbiota intervenes in the formation of these end-products from plant polysaccharides catabolism, which are then absorbed and delivered to the liver, where they are assembled in more structured lipids [2][3][4].

All abnormalities in intestinal flora taxonomic composition and/or function are usually referred to as 'dysbiosis,' a condition that has been largely explored in rodents and in NAFLD patients $[2][3][4]$. The dietary habits along with the caloric intake may strikingly contribute to the inter-individual variability of the intestinal bacterial strains. Indeed, a diet composition unbalanced in animal fat and sugars may more strongly increase the personal susceptibility to pathogenic bacteria over-growth, exerting a detrimental effect on the immunological tolerance of mucosal cells, as shown in a large number of preclinica|[5][6] and clinical studies[7][8]. In particular, Western diet and HFD have been related to the increased amount of proinflammatory bacterial species, altering gut barrier integrity, intestinal pH and lipopolysaccharide (LPS) transition into the blood flow (endotoxemia) [9]. Indeed, the intestinal barrier is constituted by tight and adherent junctions and desmosomes which hold together the epithelial cells and regulate the bidirectional flux between the gut and the liver. Specifically, intestinal barrier protects the host from pathogen invasions and impedes microbial systemic translocation [3]. Therefore, the erosion of the protective mucus layer, the reduction of antimicrobial mediators and microbial dysbiosis have been correlated with viable pathogenic bacteria, Gram-negative microbial products and pro-inflammatory luminal metabolites translocation into the blood circulation, contributing to liver damage[10][11]. The increased concentration of circulating LPS leads to the activation of Toll-like receptor 4 (TLR4)/nuclear factor kappa-light-chain-enhancer of activated B cells (NF-KB) signaling, which drives, in turn, pro-inflammatory cytokines release, reactive oxygen species (ROS) production and oxidative stress. This event cascade may trigger the activation of resident macrophages, the Kupffer cells (KCs) and Hepatic stellate cells (HSCs), further corroborating inflammation and fibrosis into the liver [12][13]. Moreover, several endogenous molecules, such as ethanol, ammonia and acetaldehyde, whose circulating increased levels result from dysbiotic microbiota (i.e., Escherichia coli abundance), are able to stimulate hepatic KCs to produce pro-inflammatory cytokines with similar mechanisms occurring in alcohol-induced liver damage [3][14].

An impairment in gut barrier function and a decrease in SCFA-producing agents have also been observed after a chronic fructose consumption, which foster macrophage activation in the liver through TLRs and Myeloid differentiation factor 88 (Myd88)-dependent pro-inflammatory pathways in mice [15]. Similarly, acute and chronic high fructose administration exacerbated endotoxemia in pediatric NAFLD patients and correlated with liver inflammation [16].

Dietary modifications can rapidly normalize intestinal microbiota, thus representing a simple and effective approach to restore eubiosis. Indeed, the diet is enabled to profoundly reshape the microbiota composition within a few hours. People consuming a Western diet and subjects with high-fiber dietary habits display a tremendous difference in microflora taxonomic composition, as shown in an elegant study in which American volunteers were randomized to receive an animal-based diet (meats, eggs and cheese) or a plant-based diet (cereals, legumes, fruits and vegetables). In individuals under animal-based regimen, an increase in bile-tolerant species such as Alistipes, Bilophila and Bacteroides and a reduction of Roseburia, Eubacterium rectale and Ruminococcus bromii, which metabolizes dietary plant polysaccharides, was observed [17][18]. In this context, a diet supplemented in fibers may favor enormous benefits for health as a consequence of their intestinal fermentation into SCFAs, mediated by colonic bacteria. Indeed, fecal samples of vegan and vegetarian individuals showed a significant decrease in Bidifobacterium and Bacteroides species, as well as Africans fed a high-carbohydrate vegetarian diet compared to English subjects assuming a mixed Western diet [19][20]. Nonetheless, a diet enriched in fibers and carbohydrates lowered fecal $\mathrm{pH}$, mainly due to the products of gut fermentative metabolism and the hampered growth of pathogens along with Escherichia coli and Enterobacteriacee [21][22].

Finally, natural extracts, such as polyphenols provided by coffee, green tea and chocolate, have been demonstrated to induce beneficial effects by directly interacting with gut microbial communities. In C57BI/6 mice fed HFD, grape polyphenols administration improved insulin sensitivity, attenuated inflammation and ameliorated intestinal barrier integrity. Overall, diets enriched in phenols have been associated with improved MetS 
features and immune tolerance, and with the restoration of intestinal barrier function, by promoting eubiosis.

\section{Changes in Gut Microbiota Species Alters the Hepatic Epigenetic Landscape: A Tipping Point}

It is a common knowledge that nutrition strongly influences microbiome composition, but it is less known that there is a long-term and persistent impact of intestinal bacteria changes on hepatic epigenome, a mechanism termed the "priming effect." Recently, Kim et al. reported that mice feeding either HFD or a high-fructose diet (HFrD) increased serum cholesterol, developed hepatic steatosis and showed an enrichment in the Odoribacter, which produces butyrate. When both HFD and HFrD groups were exposed to normal chow (NC), mice rescued only certain pathological features, such as lower body weight, improved glucose tolerance and reduced fat accumulation in the liver. Interestingly, Odoribacter remained enriched after NC and high levels of butyrate were correlated to persistent changes in liver DNA methylation, probably due to butyrate ability to act as $\mathrm{CpG}$ islands modifier, thus, suggesting a possible link between microbial by-products and nutriepigenomics [23][24].

Kimberly et al. had demonstrated that the composition of gut microbiota altered global histone acetylation and methylation in host tissues in a dietdependent manner. Mice fed a high-fat/high-sucrose (HF/HS) diet, which contains low levels of fermentable complex polysaccharides, showed loss of cecal SCFAs production (acetate, propionate and butyrate) compared to NC mice[25][26]. The reduction of SCFAs correlated to profound posttranslational modification of hepatic histones, such as lower methylation of $\mathrm{H} 3$ histones in specific aminoacidic position (H3K27me1 and H3K36me2)[25][26]. The impact of unhealthy dietary habits on NAFLD and their interaction with inherited and acquired risk factors is schematically represented in Figure 1.

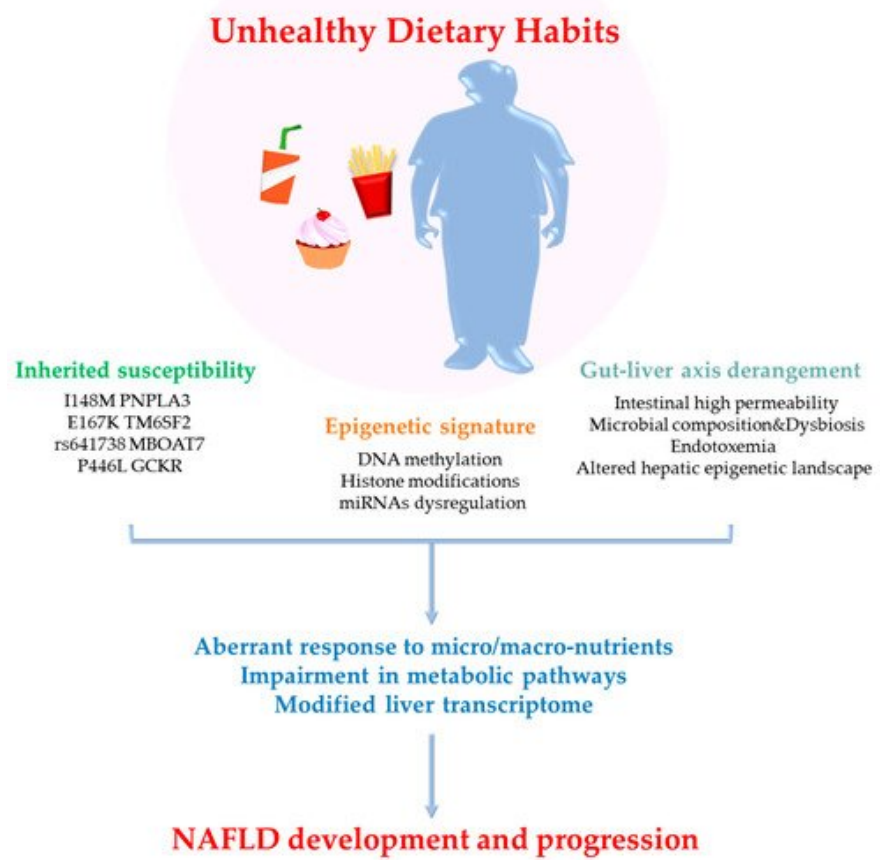

Figure 1. Nutrition, genetic and epigenetic crosstalk in nonalcoholic fatty liver disease (NAFLD).

Bioactive compounds contained in food actively contribute to physical welfare and could provide benefits as preventive and healing molecules for metabolic disorders, such as obesity, T2D and NAFLD. Conversely, unhealthy dietary habits could affect metabolic homeostasis acting at multiple levels. Chronic exposure to high fat/carbohydrate diets could increase intestinal barrier permeability, favor dysbiosis and raise the amount of circulating bacterial endotoxins, thus, unbalancing gut-liver axis bidirectional flux of metabolites. Interestingly, several micro/macro-nutrients may specifically enrich certain bacterial species, such as Odoribacter, which are involved in changes of the hepatic epigenetic landscape. In the liver, the presence of genetic variants predisposing to NAFLD development and progression (I148M PNPLA3, E167K TM6SF2, the rs641738 in MBOAT7 gene, the P446L GCKR and others) may alter the effectiveness of beneficial nutrients or accelerate the effects of unhealthy by-products derived from junk food. Similarly, epigenetic modifiers (DNA methylation, histone modifications and miRNAs regulation) could be highly nutrient-sensing, as they respond to environmental cues and could establish long-lasting effects from the gestational and post-natal period onward. The high complex network made by the combination of the environment, genes and epigenetics may cause aberrant downstream responses to nutrients, determining NAFLD onset and progression.

\section{References}

1. Ethan Hillman; Hang Lu; Tianming Yao; Cindy H. Nakatsu; Microbial Ecology along the Gastrointestinal Tract. Microbes and Environments 2017, 32, 300-313, 10.1264/jsme2.me17017.

2. Marica Meroni; Miriam Longo; Alice Rustichelli; Paola Dongiovanni; Nutrition and Genetics in NAFLD: The Perfect Binomium. International Journal of Molecular Sciences 2020, 21, 2986, 10.3390/ijms21082986.

3. Marica Meroni; Miriam Longo; Paola Dongiovanni; Alcohol or Gut Microbiota: Who Is the Guilty?. International Journal of Molecular Sciences 2019, 20, 4568, 10.3390/ijms20184568.

4. Marica Meroni; Miriam Longo; Paola Dongiovanni; The Role of Probiotics in Nonalcoholic Fatty Liver Disease: A New Insight into Therapeutic Strategies.. Nutrients 2019, 11, 2642, 10.3390/nu11112642.

5. Margarita Martinez-Medina; Jérémy Denizot; Nicolas Dreux; Frédéric Robin; Elisabeth Billard; Richard Bonnet; A Darfeuille-Michaud; 
Nicolas Barnich; Western diet induces dysbiosis with increasedE coliin CEABAC10 mice,alters host barrier function favouring AIEC colonisation. Gut 2014, 63, 116-124, 10.1136/gutjnl-2012-304119.

6. Allison Agus; Jérémy Denizot; Jonathan Thévenot; Margarita Martinez-Medina; Sébastien Massier; Pierre Sauvanet; Annick Bernalier-Donadille; Sylvain Denis; Paul Hofman; Richard Bonnet; et al.Elisabeth BillardNicolas Barnich Western diet induces a shift in microbiota composition enhancing susceptibility to Adherent-Invasive E. coli infection and intestinal inflammation.. Scientific Reports 2016, 6, 19032, 10.1038/srep19032.

7. Rasnik K. Singh; Hsin-Wen Chang; Di Yan; Kristina M. Lee; Derya Ucmak; Kirsten Wong; Michael Abrouk; Benjamin Farahnik; Mio Nakamura; Tian Hao Zhu; et al.Tina BhutaniWilson Liao Influence of diet on the gut microbiome and implications for human health.. Journal of Translational Medicine 2017, 15, 73, 10.1186/s12967-017-1175-y.

8. Jr. Ronald D. Hills; Benjamin A. Pontefract; Hillary R. Mishcon; Cody A. Black; Steven Sutton; Cory R. Theberge; Gut Microbiome: Profound Implications for Diet and Disease.. Nutrients 2019, 11, 1613, 10.3390/nu11071613.

9. L. K. Brahe; A. Astrup; L. H. Larsen; Is butyrate the link between diet, intestinal microbiota and obesity-related metabolic diseases?. Obesity Reviews 2013, 14, 950-959, 10.1111/obr.12068.

10. Göran Lindstedt; Sven Lindstedt; Bengt E. Gustafsson; Mucus in Intestinal Contents of Germfree Rats. Journal of Experimental Medicine 1965, 121, 201-213, 10.1084/jem.121.2.201.

11. Lirui Wang; Derrick E. Fouts; Peter Starkel; Phillipp Hartmann; Peng Chen; Cristina Llorente; Jessica DePew; Kelvin Moncera; Samuel B. Ho; David A. Brenner; et al.Lora V. HooperBernd Schnabl Intestinal REG3 Lectins Protect against Alcoholic Steatohepatitis by Reducing Mucosa-Associated Microbiota and Preventing Bacterial Translocation.. Cell Host \& Microbe 2016, 19, 227-39, 10.1016/j.chom.2016.01.003.

12. Shashi Bala; Miguel Marcos; Karen Kodys; Timea Csak; Nna Catalano; Pranoti Mandrekar; Gyongyi Szabo; Up-regulation of MicroRNA-155 in Macrophages Contributes to Increased Tumor Necrosis Factor $\alpha$ (TNF $\alpha$ ) Production via Increased mRNA Half-life in Alcoholic Liver Disease*. Journal of Biological Chemistry 2011, 286, 1436-1444, 10.1074/jbc.M110.145870.

13. István Hritz; Pranoti Mandrekar; Arumugam Velayudham; Donna Catalano; Angela Dolganiuc; Karen Kodys; Evelyn Kurt-Jones; Gyongyi Szabo; The critical role of toll-like receptor (TLR) 4 in alcoholic liver disease is independent of the common TLR adapter MyD88.. Hepatology 2008, 48, 1224-31, 10.1002/hep.22470.

14. Lixin Zhu; Susan S. Baker; Chelsea Gill; Wensheng Liu; Razan Alkhouri; Robert D. Baker; Steven R. Gill; Characterization of gut microbiomes in nonalcoholic steatohepatitis (NASH) patients: A connection between endogenous alcohol and NASH. Hepatology 2013, 57, 601-609, 10.1002/hep.26093.

15. Sabine Wagnerberger; Astrid Spruss; Giridhar Kanuri; Valentina Volynets; Carolin Stahl; Stephan Bischoff; Ina Bergheim; Toll-like receptors 1-9 are elevated in livers with fructose-induced hepatic steatosis. British Journal of Nutrition 2012, 107, 1727-1738, 10.1017/s0007114511004983.

16. Ran Jin; Andrew Willment; Shivani S. Patel; Xiaoyan Sun; Ming Song; Yanci O. Mannery; Astrid Kosters; Craig J. McClain; Miriam Vos; Fructose Induced Endotoxemia in Pediatric Nonalcoholic Fatty Liver Disease. International Journal of Hepatology 2014, 2014, 560620, 10.1155/2014/560620.

17. Shin Yoshimoto; Tze Mun Loo; Koji Atarashi; Hiroaki Kanda; Seidai Sato; Seiichi Oyadomari; Yoichiro Iwakura; Kenshiro Oshima; Hidetoshi Morita; Masahira Hattori; et al.Kenya HondaYuichi IshikawaEiji HaraNaoko Ohtani Obesity-induced gut microbial metabolite promotes liver cancer through senescence secretome. Nature 2013, 499, 97-101, 10.1038/nature12347.

18. Suzanne Devkota; Yunwei Wang; Mark W. Musch; Vanessa Leone; Hannah Fehlner-Peach; Anuradha Nadimpalli; Dionysios A. Antonopoulos; Bana Jabri; Eugene B Chang; Dietary-fat-induced taurocholic acid promotes pathobiont expansion and colitis in II10-/- mice. Nature 2012, 487, 104-8, 10.1038/nature11225.

19. J Zimmer; B Lange; Julia-Stefanie Frick; H Sauer; K Zimmermann; Andreas Schwiertz; K Rusch; S Klosterhalfen; P Enck; A vegan or vegetarian diet substantially alters the human colonic faecal microbiota. European Journal of Clinical Nutrition 2012, 66, 53-60, 10.1038/ejcn.2011.141.

20. V Aries; J S Crowther; B S Drasar; M J Hill; R E Williams; Bacteria and the aetiology of cancer of the large bowel.. Gut 1969, 10, 334-335, 10.1136/gut.10.5.334.

21. Michael Conlon; Anthony R. Bird; The Impact of Diet and Lifestyle on Gut Microbiota and Human Health. Nutrients 2014, 7, 17-44, 10.3390/nu7010017.

22. Christophe Chassard; Christophe Lacroix; Carbohydrates and the human gut microbiota. Current Opinion in Clinical Nutrition and Metabolic Care 2013, 16, 453-460, 10.1097/mco.0b013e3283619e63.

23. Umesh Wankhade; Ying Zhong; Ping Kang; Maria Alfaro; Sree V. Chintapalli; Keshari M. Thakali; Kartik Shankar; Enhanced offspring predisposition to steatohepatitis with maternal high-fat diet is associated with epigenetic and microbiome alterations. PLOS ONE 2017, 12, e0175675, 10.1371/journal.pone.0175675.

24. Hyun-Jung Kim; Suk-Chul Bae; Histone deacetylase inhibitors: molecular mechanisms of action and clinical trials as anti-cancer drugs. American journal of translational research 2011, 3, 166-179.

25. Yufeng Qin; Paul A Wade; Crosstalk between the microbiome and epigenome: messages from bugs.. The Journal of Biochemistry 2018, 163, 105-112, 10.1093/jb/mvx080.

26. Kimberly Krautkramer; Julia H. Kreznar; Kymberleigh A. Romano; Eugenio I. Vivas; Gregory A. Barrett-Wilt; Mary E. Rabaglia; Mark P. Keller; Alan D. Attie; Federico E Rey; John M. Denu; et al. Diet-Microbiota Interactions Mediate Global Epigenetic Programming in 
Multiple Host Tissues.. Molecular Cell 2016, 64, 982-992, 10.1016/j.molcel.2016.10.025.

\section{Keywords}

nonalcoholic fatty liver disease; nonalcoholic steatohepatitis; NAFLD; NASH; gut microbiota; gut-liver axis; leaky gut; intestinal permeability; nutrition; nutriepigenomics.

(c) (i) (C) 2020 by the author(s). Distribute under a Creative Commans CC BY license 